\title{
Improvement of the Quasi-Yagi Antenna Perfomances by Using an Ends-fed Dipole Driver
}

\author{
Dmitriy A. Buhtiyarov, Anatoly P. Gorbachev", Svetlana Yu. Zhelezko \\ Radio Received and Transmitted Device Department, Novosibirsk State Technical University, Novosibirsk, 630092, Russia \\ *Corresponding Author: apgor@ngs.ru
}

Copyright (C) 2014 Horizon Research Publishing All rights reserved.

\begin{abstract}
A novel approach to improve the quasi-Yagi antenna performances by using the so-called ends-fed dipole radiator is presented. To illustrate this idea a cylindrical prototype operating at $790 \mathrm{MHz}$ is developed and tested. Since the area around the radiating arms is free of any conductors, the proposed radiator is a good candidate to create high polarization purity of radiation. The measured results have revealed that such a driver is able to achieve an effective area reduction to $20 \%$ with enhanced up to $17 \mathrm{~dB}$ front-to-back ratio without decreasing the bandwidth.
\end{abstract}

Keywords Cylindrical Radiator, Current Distribution, Induced Emf Method, Quasi-Yagi Antenna

\section{Introduction}

Modern wireless communication systems need the antennas to be compact as possible. A suppression of an unwanted radio frequency emission has led to an increasing demand for directional antennas such as quasi-Yagi arrays. There have been many Yagi-Uda/quasi-Yagi antennas with a strip/coaxial feed and a dipole driver that have been presented over the last ten years [1-4]. Each of these antennas is the successful discovery in terms of its bandwidth, radiation patterns, size reduction, and simplify of manufacturing. However, all the antennas contain a balun that is used to excite the center-fed dipole driver. Frequently, the $180^{\circ}$ out-of-phase balun nodes are displaced in the area substantially that increases the surroundings around the dipole nodes and affects polarization purity of radiation. This also needs accurate accomplishment of influence design including the length of the line as well as the meandering/turning of that.

Below we propose a modified dipole-like radiator. Its architecture is the result of an attempt to alleviate the complication of a coaxial/strip feed with a regular wire antenna design principle by combining the nonstandard ends-fed dipole radiator (EFDR) and an usual balun such as coupled line directional coupler or branch-line one. After its careful design, this EFDR being assembled with corresponding ground exteriors (such as ground planes of a balun) acts as a directive standalone antenna or plays the role of a radio frequency illuminator in a quasi-Yagi assembly.

An analytical study of the EFDR is presented, the numerical optimization of the quasi-Yagi antenna by proper 3D EM solver is described, and experimental results given in the Section 4 are used to verify the proposed approach.

\section{Current Distribution, Radiated Fields, and Radiation Resistance of the EFDR}

An ends-fed dipole radiator contains two collinear cylindrical wires 1 and 2 , an energized coaxial cable 3 , and a balun 4 having the input port 5 and $180^{\circ}$ out-of-phase output ports 6 and 7 (Fig. 1).

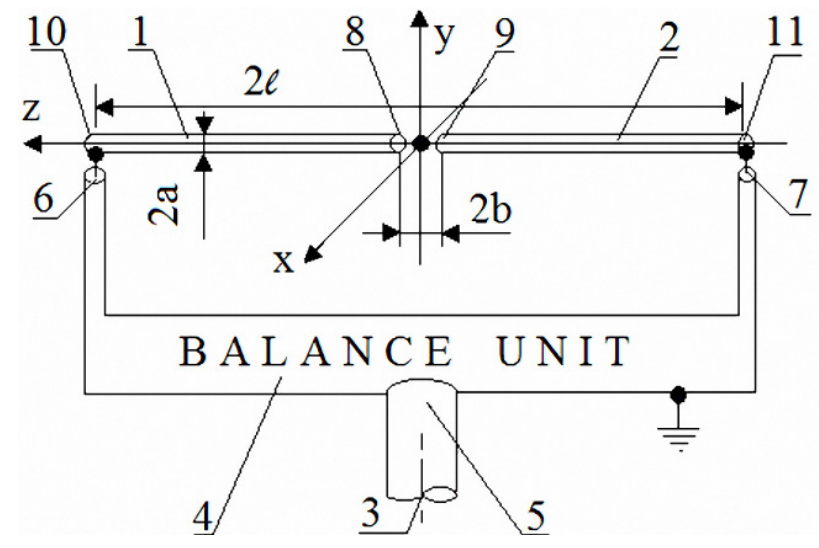

Figure 1. An ends-fed dipole radiator

The following analysis applies to both perfectly-conducting wires 1 and 2 of radii $a$ that are electrically thin $(a \ll \lambda)$, where $\lambda$ is the free-space wavelength. The adjacent ends 8 and 9 are placed to produce the immediate nearness $(b \ll \lambda)$, and the $180^{\circ}$ out-of phase outputs 6 and 7 are connected to the far ends 10 and 11 of collinear wires, respectively. Both the wires each of length $l$ are centered and oriented along the $z$ axis. The surroundings of the EFDR are the homogeneous outside 
space characterized by permittivity $\varepsilon$ and permeability $\mu$ with corresponding wave number $k=\omega \sqrt{\varepsilon \mu}$ and intrinsic impedance $\eta=\sqrt{\mu / \varepsilon}$.

In order to illustrate the creation of the current distribution on the EFDR, let us first begin according to [5, Chapter 1] with the geometry of an open-circuited lossless two-wire balanced (symmetrical) transmission line as shown in Fig.2A.
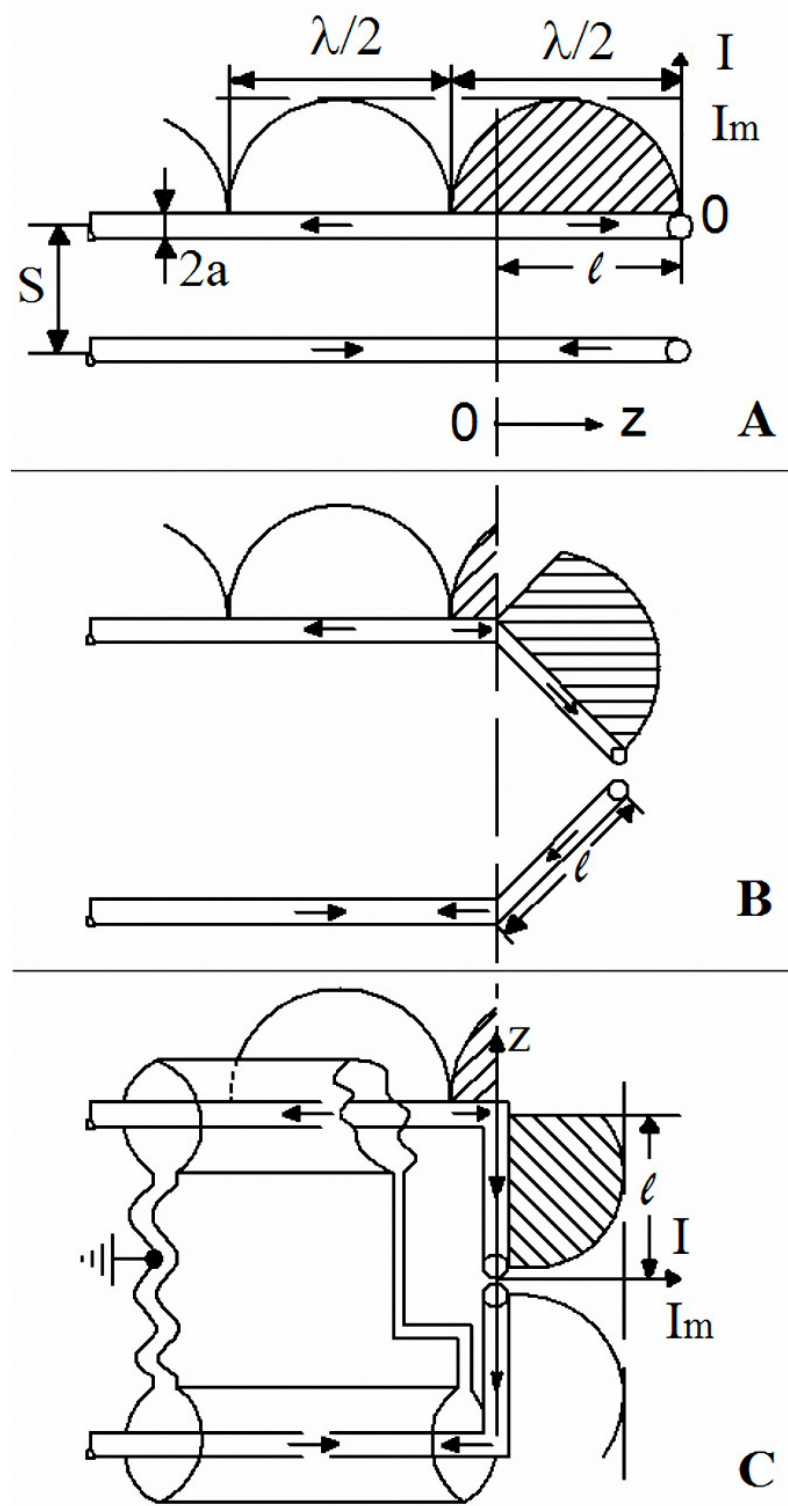

Figure 2. Current distribution on (A) a lossless open-circuited two-wire transmission line, (B) tilted transmission line, and (C) a linear ends-fed dipole radiator

For this line the current in a half-cycle of one wire is of the same magnitude but $180^{\circ}$ out-of-phase from that in the corresponding half-cycle of the other wire. If in addition the spacing between the two wires is very small $(s \ll \lambda)$ or both the wires are placed inside the ground hollow pipe, the fields radiated by the current of each wire are essentially cancelled by those of the other.
As the section of the transmission line outside the hollow pipe between $0 \leq z \leq l$ begins to tilt as shown in Fig. 2B, it can be assumed that the current distribution is essentially unaltered in form in each of the wires [6]. However, because the two wires of the tilted section are not necessarily close to each other, the fields radiated by one do not necessarily cancel those of the other. Ultimately the tilted section of the transmission line can take the form shown in Fig. 2C. If the cross-section of the hollow pipe is the figure-dumb-bell with very thin cross-piece rather than the circle, then this is the geometry of the proposed linear EFDR. If the diameter of each wire is very small, the ideal standing wave pattern of the current along the arms of the EFDR is sinusoidal with a null at the end. Since both the ends of the wires are placed almost in the origin, the current distribution can be written as

$$
I\left(x^{\prime}=0, y^{\prime}=0, z^{\prime}\right)=\left\{\begin{array}{l}
+I_{m} \sin k z^{\prime}, 0 \leq z^{\prime} \leq l \\
-I_{m} \sin k z^{\prime},-l \leq z^{\prime} \leq 0
\end{array}\right.
$$

where $\left(x^{\prime}, y^{\prime}, z^{\prime}\right)$ represent the coordinates of the radiator.

The following analysis is based on the approach described in [5, Chapter 4]. The finite radiator of Fig. 1 is subdivided into a number of infinitesimal dipoles. For such a dipole positioned along the $z$ axis at $z^{\prime}$, the electric field component in the far-field region is given as

$$
d E_{\theta}=j \eta \frac{k I\left(z^{\prime}\right) e^{-j k R}}{4 \pi R} \sin \theta d z^{\prime}
$$

where $R$ is a distance from any point on the radiator to the observation point which is characterized by radial distance $r$. Using corresponding far-field approximations, (2) can be written as

$$
d E_{\theta}=j \eta \frac{k I\left(z^{\prime}\right) e^{-j k r}}{4 \pi r} \sin \theta e^{+j k z^{\prime} \cos \theta} d z^{\prime}
$$

Summing the contributions from all the elements, we can write for the distribution of (1)

$$
\begin{gathered}
E_{\theta}=\int_{-l}^{l} d E_{\theta}=j \eta \frac{k e^{-j k r}}{4 \pi r} \sin \theta \\
\times\left[\int_{-l}^{0} \sin (-k z) e^{\left.j k z^{\prime} \cos \theta_{d z^{\prime}}+\int_{0}^{l} \sin \left(k z^{\prime}\right) e^{j k z^{\prime} \cos \theta_{d z^{\prime}}}\right]}\right.
\end{gathered}
$$

After corresponding manipulations, (4) takes the form of

$$
\begin{gathered}
E_{\theta}=j \eta[Q / \sin \theta] I_{m} e^{-j k r} / 2 \pi r \\
Q=1-\cos (k l \cos \theta) \cos k l-\sin (k l \cos \theta) \sin k l \cos \theta
\end{gathered}
$$

Next, the average Poynting vector $\boldsymbol{W}_{a v}$ is given by

$$
\boldsymbol{W}_{\boldsymbol{a} \boldsymbol{v}}=\frac{1}{2} \operatorname{Re}\left[\boldsymbol{E} \times \boldsymbol{H}^{*}\right]=\boldsymbol{r}_{\boldsymbol{0}} \frac{1}{2 \eta}\left|E_{\theta}\right|^{2}
$$

To find the total power radiated $P_{r a d}$, the average Poynting vector of (7) is integrated over a sphere of radius $r$. Thus, for a free-space ( $\eta=120 \pi \mathrm{Ohms})$ 


$$
\begin{gathered}
P_{\text {rad }}=\oiint\left(\boldsymbol{W}_{\boldsymbol{a} \boldsymbol{v}} \cdot \boldsymbol{d s}\right)=\int_{0}^{2 \pi} \int_{0}^{\pi} W_{a v} r^{2} \sin \theta d \theta d \varphi \\
=30 I_{m}^{2} \int_{0}^{\pi} \frac{Q^{2}}{\sin \theta} d \theta
\end{gathered}
$$

The radiation resistance $R_{r}$ can be obtained using $P_{\text {rad }}=\left|I_{m}\right|^{2} R_{r} / 2$ and (8), and can be written as (please see Appendix I)

$$
\begin{gathered}
R_{r}=2 P_{\text {rad }} /\left|I_{m}\right|^{2}=60 \int_{0}^{\pi}\left[Q^{2} / \sin \theta\right] d \theta \\
=30\left\{3 \gamma+\ln \left[4(k l)^{3}\right]+C i(4 k l)-4 C i(2 k l)\right. \\
\left.+2 \sin (k l)^{2}\left[\frac{\sin (2 k l)}{2 k l}-1\right]\right\}
\end{gathered}
$$

where $\gamma=0.5772157$ (Euler's constant) and $C i(x)$ is the cosine integral.

Now, according to [5, Chapter 8] the real part $R_{m}$ of an input impedance $Z_{m}=R_{m}+j X_{m}$ referred to at the current maximum $I_{m}$ can be expressed as $R_{m}=R_{r}$ whereas the input resistance $R_{i n}$ referred to at the current $I_{l}$ at the input terminals 10 and 11 (Fig. 1) can be obtained by a transfer relation $I_{l}=I_{m} \sin (k l)$, or

$$
R_{\text {in }}=R_{m} / \sin (k l)^{2}
$$

\section{Self-impedance of the EFDR}

The induced emf method leads to closed-form solutions which provide such the design data that can be used directly as very good initial parameters in full-wave 3D EM simulation. When the input reactance is desired, the near-fields must be known. These fields are derived based on the geometry of Fig. 3.

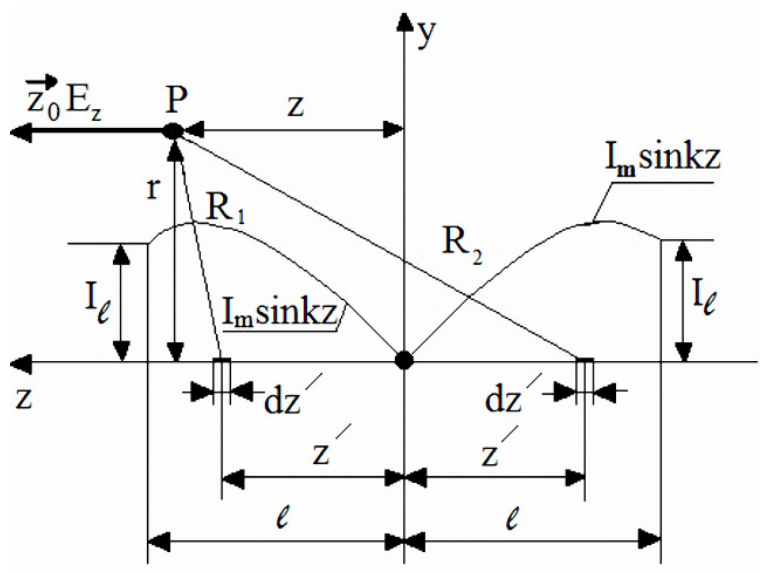

Figure 3. A geometrical arrangement of the EFDR for the near-field approximation
The following procedure be like to that used in [5] for the classical dipole. Since the EFDR carries an electric current $I\left(z^{\prime}\right)$ only, both magnetic current and related potential function are zero. To find the vector potential $\boldsymbol{A}(x, y, z)$ generated by a given electric current density $\boldsymbol{J}=\boldsymbol{x}_{\boldsymbol{0}} J_{x}+\boldsymbol{y}_{\boldsymbol{0}} J_{y}+\boldsymbol{z}_{\boldsymbol{0}} J_{z} \approx \boldsymbol{z}_{\boldsymbol{0}} J_{z}\left(z^{\prime}\right)$ we write

$$
\boldsymbol{A} \approx \boldsymbol{z}_{\boldsymbol{0}} A_{z}(x, y, z)=\boldsymbol{z}_{\boldsymbol{0}} \frac{\mu}{4 \pi} \int_{-l}^{+l} 2 \pi a J_{z}(z) \frac{e^{-j k R}}{R} d z^{\prime}
$$

For a cylindrical wire, the total current $I\left(z^{\prime}\right)$ is uniformly distributed around the surface of the wire and it forms a linear current sheet with an electric density $J_{z}\left(z^{\prime}\right)$. The current is concentrated primarily over a very small thickness of the conductor and is given by $I\left(z^{\prime}\right)=2 \pi a J_{z}\left(z^{\prime}\right)=I_{m} \sin \left(k z^{\prime}\right)$. Substituting (1) into (11) leads to the formula

$$
\begin{aligned}
& A_{z}(x, y, z)=\frac{\mu}{4 \pi} \int_{-l}^{l} I(z) \frac{e^{-j k R}}{R} d z \\
& =\frac{\mu}{4 \pi} \int_{0}^{l} I\left(z^{\prime}\right)\left[\frac{e^{-j k R_{1}}}{R_{1}}+\frac{e^{-j k R_{2}}}{R_{2}}\right] d z^{\prime}
\end{aligned}
$$

where the integration performs from $z^{\prime}=0$ until $z^{\prime}=l$ since the currents in the wires are symmetrical with respect to the origin. The infinitesimal dipoles positioned along the z-axis at $\pm z^{\prime}$ are removed from the point $P$ (Fig. 3) at the distances

$$
R_{1}=\sqrt{r^{2}+\left(z-z^{\prime}\right)^{2}}, R_{2}=\sqrt{r^{2}+\left(z+z^{\prime}\right)^{2}}
$$

The electric field z-component $E_{z}$ can now be found using the expression [5, page 136]

$$
\boldsymbol{E}=-j \omega \boldsymbol{A}-j \frac{1}{\omega \mu \varepsilon} \nabla(\nabla \cdot \boldsymbol{A})
$$

Substituting (13) into (12) and taking into account the coupling between partial derivatives

$$
\frac{\partial^{2} R_{1}}{\partial z^{2}}=\frac{\partial^{2} R_{1}}{\partial z^{2}}, \frac{\partial^{2} R_{2}}{\partial z^{2}}=\frac{\partial^{2} R_{2}}{\partial z^{2}}
$$

we can write the following relationship

$$
\begin{gathered}
E_{z}=-j \frac{\omega \mu}{4 \pi} \int_{0}^{l} I\left(z^{\prime}\right) \frac{e^{-j k R}}{R} d z^{\prime}-j \frac{1}{4 \pi \omega \varepsilon} \\
\times \frac{\partial^{2}}{\partial z^{2}}\left[\int_{0}^{l} I\left(z^{\prime}\right) \frac{e^{-j k R}}{R} d z^{\prime}\right]=-\frac{1}{4 \pi \omega \varepsilon} \int_{0}^{l}\left\{I ( z ^ { \prime } ) \frac { \partial ^ { 2 } } { \partial z ^ { 2 } } \left[\frac{e^{-j k R_{1}}}{R_{1}}\right.\right.
\end{gathered}
$$




$$
\begin{gathered}
\left.\left.+\frac{e^{-j k R_{2}}}{R_{2}}\right]+k^{2} I\left(z^{\prime}\right)\left[\frac{e^{-j k R_{1}}}{R_{1}}+\frac{e^{-j k R_{2}}}{R_{2}}\right]\right\} d z^{\prime} \\
=-\frac{1}{I_{m}} \int_{-l}^{l} \sin \left(k z^{\prime}\right) E_{z}\left(r=a, z=z^{\prime}\right) d z^{\prime}
\end{gathered}
$$

Two-step integration by parts in (15) yields

$$
\begin{gathered}
E_{z}=-j \frac{1}{4 \pi \omega \epsilon}\left\{\left.I\left(z^{\prime}\right) \frac{d}{d z^{\prime}}\left[\frac{e^{-j k R_{1}}}{R_{1}}+\frac{e^{-j k R_{2}}}{R_{2}}\right]\right|_{z^{\prime}=0} ^{z^{\prime}=l}\right. \\
-\left.\frac{d I\left(z^{\prime}\right)}{d z^{\prime}}\left[\frac{e^{-j k R_{1}}}{R_{1}}+\frac{e^{-j k R_{2}}}{R_{2}}\right]\right|_{z^{\prime}=0} ^{z^{\prime}=l} \\
\left.+\int_{0}^{l}\left[\frac{d^{2} I\left(z^{\prime}\right)}{d z^{2}}+k^{2} I\left(z^{\prime}\right)\right]\left[\frac{e^{-j k R_{1}}}{R_{1}}+\frac{e^{-j k R_{2}}}{R_{2}}\right] d z^{\prime}\right\} \\
=-j \frac{1}{4 \pi \omega \varepsilon}\left[\left.B_{1}\right|_{z^{\prime}=0} ^{z^{\prime}=l}-\left.B_{2}\right|_{z^{\prime}=0} ^{z^{\prime}=l}+B_{3}\right]
\end{gathered}
$$

where

$$
\begin{aligned}
&\left.B_{1}\right|_{z ?=} ^{z^{\prime}=l}= I_{m} \sin (k l)\left[\frac{e^{-j k R_{1} l}}{R_{1 l}^{3}}(z-l)\left(1+j k R_{1 l}\right)\right. \\
&\left.-\frac{e^{-j k R_{2 l} l}}{R_{2 l}^{3}}(z+l)\left(1+j k R_{2 l}\right)\right] \\
&\left.B_{2}\right|_{z^{\prime} ?=} ^{z^{\prime}=l}=k I_{m}\left\{\cos (k l)\left[\frac{e^{-j k R_{1 l}}}{R_{1 l}}+\frac{e^{-j k R_{2 l} l}}{R_{2 l}}\right]-2 \frac{e^{-j k R_{0}}}{R_{0}}\right\} \\
& R_{1 l}= \sqrt{r^{2}+(z-l)^{2}}, R_{2 l}=\sqrt{r^{2}+(z+l)^{2}}, \\
& R_{0}=\sqrt{r^{2}+z^{2}}
\end{aligned}
$$

The third quantity $B_{3}$ in (16) vanishes because the factor $\left[d^{2} I\left(z^{\prime}\right) / d z^{\prime 2}+k^{2} I\left(z^{\prime}\right)\right]$ is equal to zero at all the points of integration along the radiator.

Using the current distribution of (1) and tangential electric field of (15), the input impedance referred to at the current maximum $I_{m}$ is defined as [5, page 465]

$$
Z_{m}=-\frac{1}{I_{m}^{2}} \int_{-l}^{l} I\left(r=a, z=z^{\prime}\right) E_{z}\left(r=a, z=z^{\prime}\right) d z^{\prime}(17)
$$

In other words, the complex power input into radiator is equal to the complex radiated power at the surface of the wires [7]. Substituting (16) into (17) it can be shown that both the real $R_{m}\left(R_{i n}\right)$ and the imaginary $X_{m}\left(X_{i n}\right)$ part of the self- (input) impedance $Z_{m}=R_{m}+j X_{m}$ referred to at the current maximum (at the current $I_{l}$ at the input terminals) can be expressed for a free-space as (in Ohms)

$$
Z_{m}=30 \int_{0}^{l}\left\{\frac { \operatorname { s i n } ( k l ) } { k } \left(\frac{e^{-j k R 1(z)}(z-l)(1+j k R 1(z))}{R 1^{3}(z)}\right.\right.
$$

$$
\begin{gathered}
\left.-\frac{e^{-j k R 2(z)}(z+l)(1+j k R 2(z))}{R 2^{3}(z)}\right)-\cos (k l)\left(\frac{e^{-j k R 1(z)}}{R 1(z)}\right. \\
\left.\left.+\frac{e^{-j k R 2(z)}}{R 2(z)}\right)+2 \frac{e^{-j k R 0(z)}}{R 0(z)}\right\}\left(e^{j k z}-e^{-j k z}\right) d z
\end{gathered}
$$

$$
R_{m}=60\left\{\sin ^{2}(k l)\left(\frac{\sin \left(k l \sqrt{4+(a / l)^{2}}\right)}{k l \sqrt{4+(a / l)^{2}}}-\frac{\sin (k a)}{k a}\right)+3 C i(k a)\right.
$$$$
+0.5 \operatorname{Ci}\left[k l\left(\sqrt{4+(a / l)^{2}}+2\right)\right]+0.5 \operatorname{Ci}\left[k l\left(\sqrt{4+(a / l)^{2}}-2\right)\right]
$$$$
\left.-2 C i\left[k l\left(\sqrt{1+(a / l)^{2}}+1\right)\right]-2 C i\left[k l\left(\sqrt{1+(a / l)^{2}}-1\right)\right]\right\}
$$

$$
\begin{gathered}
X_{m}=60\left\{\sin ^{2}(k l)\left(\frac{\cos \left(k l \sqrt{4+(a / l)^{2}}\right)}{k l \sqrt{4+(a / l)^{2}}}-\frac{\cos (k a)}{k a}\right)-3 \operatorname{Si}(k a)\right. \\
-0.5 \operatorname{Si}\left[k l\left(\sqrt{4+(a / l)^{2}}+2\right)\right]-0.5 \operatorname{Si}\left[k l\left(\sqrt{4+(a / l)^{2}}-2\right)\right] \\
\left.+2 \operatorname{Si}\left[k l\left(\sqrt{1+(a / l)^{2}}+1\right)\right]+2 \operatorname{Si}\left[k l\left(\sqrt{1+(a / l)^{2}}-1\right)\right]\right\} \quad(20) \\
R_{\text {in }}=R_{m} /[\sin (k l)]^{2}, X_{\text {in }}=X_{m} /[\sin (k l)]^{2}
\end{gathered}
$$

The derivation of expressions (19) and (20) is given in Appendix II. If the radius $a=0.0001 l$ then the result of (19) obtained by an induced emf method is equal to that of (9) obtained by integration over a closed sphere, namely for $l / \lambda=0.289$ we have

$$
R_{m}=94.363, R_{\text {in }}=100.264
$$

This coincidence both the quantities $R_{r}(9)$ and $R_{m}$ (19) may be considered as the mutual verification of calculations.

\section{Quasi-yagi Antenna}

The physical characteristics for the antenna presented are the same as from the conventional design reported in [5], however the corresponding arrangement is quite different 
(Fig. 4).

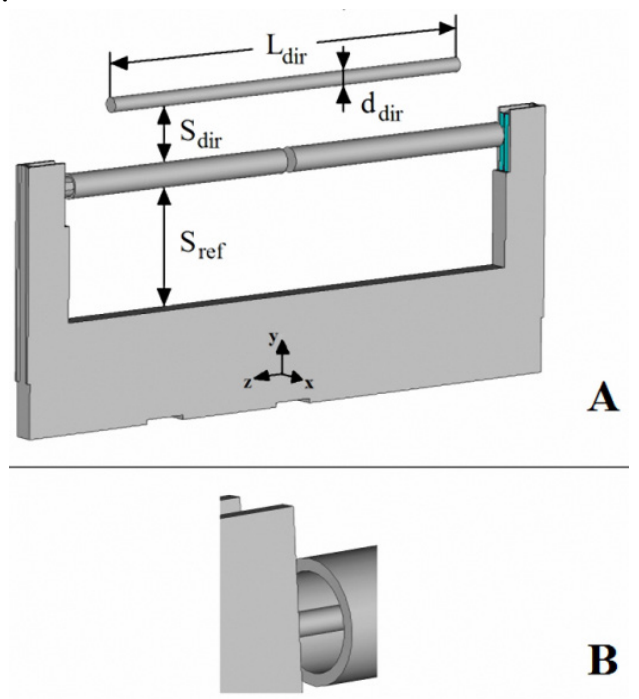

Figure 4. 3D view of the proposed quasi-Yagi antenna: (A) large scale, (B) fine scale

The presence of the ground pencil-case containing the balun affects the coupling between the director and the driver requiring that both the director ( $\left.S_{d i r}\right)$ and the reflector ( $S_{r e f}$ ) spacing be modified relative to the conventional design. The values for $S_{\text {dir }}, S_{\text {ref }}$ were initially chosen by using [5, Table 10-6] and then refined through an EM simulation in order to minimize the return loss at the input port of the balun used. Its implementation follows the classical procedure to realize the quarter-wave coupled strip-line directional coupler [8]. The required phase shift is obtained by adjusting the length difference between outgoing lines to be equal $0.5 \lambda / \sqrt{\varepsilon_{r}}$, where $\varepsilon_{r}$ is the dielectric constant of the material used to realize the coupler. Since the characteristic impedance of the coaxial cable 3 (Fig. 1) is equal to $50 \mathrm{Ohms}$ and the radiator is connected to the balun in series [9], the input resistance $R_{i n}$ should be twice ( $R_{\text {in }}=100 \mathrm{Ohms}$ ). This requires that the length of radiating wires (Fig. 1) should be chosen initially as indicated above for the parameter list (22). As follows from (20), the condition $l / \lambda=0.289$ gives the input reactance for the actual set of radii $(0.01 \leq a / l \leq 0.05)$ around $-j 650 \mathrm{Ohms}$ (the thinner the wire, the closer the reactance is to $-j 700$ Ohms). Since the balun introduces into radiator an inductive reactance $X_{b a l}$ which is a function of the distance $S_{r e f}$, only the difference around $X_{b a l}-j 650$ should be compensated by some means. To do so the inductive reactance $X^{*}=j\left|X_{b a l}-650\right| / 2$ is introduced initially in an equivalent representation of the radiator at both the arms in series [10]. This reactance is performed as the short-circuited coaxial line of length $l^{*}$ with the corresponding inner and outer radii $r^{*}$ and $R^{*}$, respectively, placed inside the hollow radiating arm, where $l^{*}<l, R^{*}<a$. The simulation/optimization by WIPL-D [11] for $\varepsilon_{r}=2.5$ (Russian dielectric material FAF-4) leads to the following final dimensions (in millimeters):

$$
\begin{gathered}
l=100, a=3, l^{*}=71, r^{*}=0.8, R^{*}=2.5, L_{d i r}=164, \\
d_{\text {dir }}=4.5, S_{\text {dir }}=21.8, S_{\text {ref }}=69
\end{gathered}
$$

A comparison between measured and simulated data is presented below.

The results for the return loss $\left(S_{11}\right)$ and isolation $\left(S_{14}\right)$ of a balun (Fig. 5) that excites the antenna show close comparison.

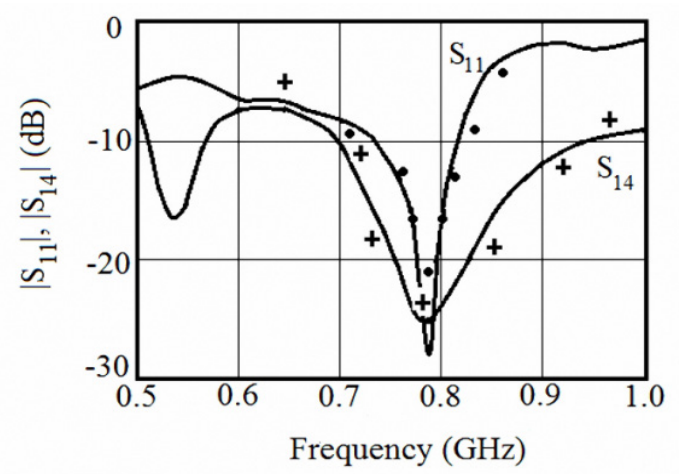

Figure 5. Simulated $(-)$ and measured $(\cdots,+++)$ return loss $\left(S_{11}\right)$ and isolation $\left(S_{14}\right)$ of a balun that excites the quasi-Yagi antenna

Some discrepancy is attributed to impedance discontinuities in the test fixture RF connector (not shown in Fig. 4) and an attainable resolution of the etching process that was used to realize the printed-circuit boards of the balun.
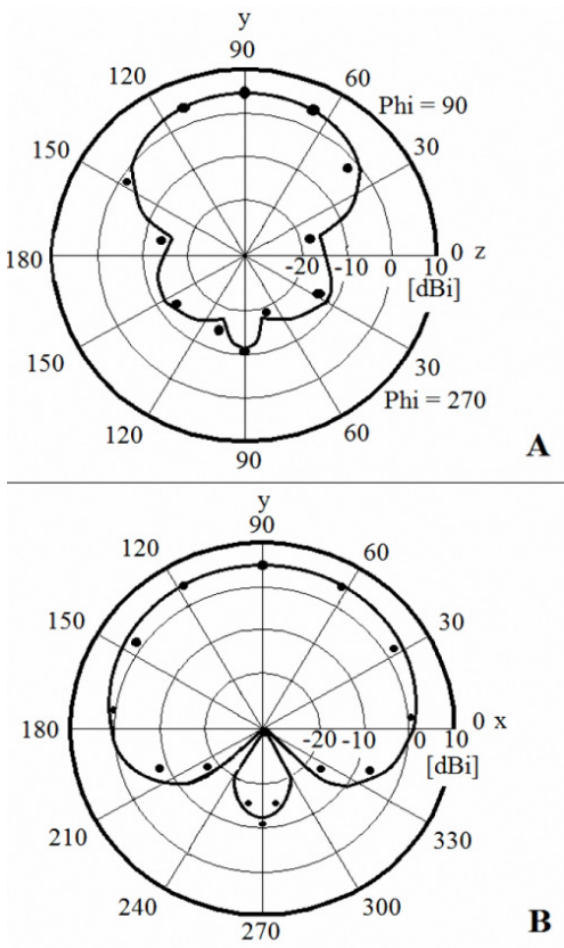

Figure 6. Simulated $(-)$ and measured $(\cdots)$ radiation patterns: (A) E-plane, (B) H-plane 
Fig. 6 shows both the measured and predicted E-plane (yz-plane cut) and H-plane (xy-plane cut) pattern at 790 $\mathrm{MHz}$ with the front-to-back ratio approximately $17 \mathrm{~dB}$. The simulated cross-polarization fields of the antenna exhibit almost omnidirectional patterns with the peak around -35 and $-26 \mathrm{dBi}$ in the $\mathrm{E}-$ and $\mathrm{H}-$ plane, respectively, and are not shown in the plots for brevity. The measured cross-polarization patterns are $6 \mathrm{~dB}$ larger than those produced through the simulation.

\section{Conclusion}

A new implementation of a quasi-Yagi antenna with end fire like radiation and good polarization purity has been proposed. To realize the necessary pattern an ends-fed dipole radiator has been investigated. These radiation properties make the radiator as a qualitative standalone module or a suitable candidate for phased arrays. An attractive feature of the antenna is its compact size for UHF band that paves the way for new applications in wireless communication systems.

\section{Appendix I}

To obtain the closed-form expression of (9), the introduction of the supplementary symbols to denote constants has been made: $\alpha=k l, s=\sin \alpha, s_{2}=\sin (2 \alpha)$, $s_{4}=\sin (4 \alpha), c=\cos \alpha, \quad c_{2}=\cos (2 \alpha), \quad c_{4}=\cos (4 \alpha)$. Let us begin with the transformation of expression (6) in the binomial:

$$
Q=-c[\cos (\alpha \cos \theta)-c]-s[\sin (\alpha \cos \theta) \cos \theta-s]
$$

Square (23), we get three terms in the numerator of integrand of the integral (9), equal to the sum of three integrals respectively:

$$
R_{r}=R 1+R 2+R 3
$$

We will calculate these three integrals by turns, and then sum them to (24). In order to shorten the text we will normalize integrals to the constants that have been obtained after squaring (23). For cancellation we will use such standard trigonometric formulas as double-angle, lowering the degree and so on. We will also use such rule as

$$
\int_{b}^{a} f(x) d x=-\int_{a}^{b} f(x) d x
$$

During the integration several changes of integration variable will be carried out. First change of variable is $x=\cos \theta, \theta=\arccos (x)$,

$$
d \theta=-d x / \sqrt{1-x^{2}}, \sin \theta=\sqrt{1-x^{2}}
$$

after recalculation of limits of integration, we get upper limit $a=\cos \pi=-1$, lower limit $b=\cos 0=1$.
Calculation of the first integral in (24) proceeds as follows, using (26) and (25) by turns:

$$
\begin{gathered}
\frac{R 1}{60 c^{2}}=\int_{0}^{\pi} \frac{[\cos (\alpha \cos \theta)-c]^{2}}{\sin \theta} d \theta \\
=\int_{1}^{-1} \frac{[\cos (\alpha x)-c]^{2}}{\sqrt{1-x^{2}}}\left(\frac{-d x}{\sqrt{1-x^{2}}}\right)=\int_{-1}^{1} \frac{[\cos (\alpha x)-c]^{2}}{1-x^{2}} d x
\end{gathered}
$$

Applying partial fraction expansion to the denominator of (27)

$$
1 /\left(1-x^{2}\right)=0.5(1 /(1-x)+1 /(1+x))
$$

together with (25), we obtain

$$
\frac{R 1}{60 c^{2}}=\int_{-1}^{1} \frac{[\cos (\alpha x)-c]^{2}}{1+x} d x
$$

Second change of variable is

$$
\begin{gathered}
u=\alpha(x+1), \quad x=(u / \alpha)-1, d x=d u / \alpha, \\
a=2 \alpha, b=0
\end{gathered}
$$

After performing (30) in (29), we obtain

$$
\begin{gathered}
\frac{R 1}{60 c^{2}}=\int_{0}^{2 \alpha} \frac{[\cos [\alpha((u / \alpha)-1)]-\mathrm{c}]^{2}}{1+(u / \alpha)-1} \frac{d u}{\alpha} \\
=-s_{2} \int_{0}^{2 \alpha} \frac{\sin u}{u} d u+\frac{s_{2}}{2} \int_{0}^{2 \alpha} \frac{\sin (2 u)}{u} d u \\
+\int_{0}^{2 \alpha} \frac{-2 c^{2} \cos u+c^{2}+c^{2}\left(1-\sin ^{2} u\right)+s^{2} \sin ^{2} u}{u} d u
\end{gathered}
$$

Integrals in (31) can't be expressed in terms of elementary functions. We will use such special functions as: sine integral

$$
\operatorname{Si}(x)=\int_{0}^{x} \frac{\sin u}{u} d u
$$

cosine integral

$$
C i(x)=\gamma+\ln (x)+\int_{0}^{x} \frac{\cos u-1}{u} d u
$$

related cosine integral

$$
C i_{n}(x)=\int_{0}^{x} \frac{1-\cos u}{u} d u=\gamma+\ln (x)-C i(x)
$$

where $\gamma=0.5772157$ (Euler's constant). Expressing (31) through (32), we obtain

$$
R 1 / 60 c^{2}=-s_{2} S i(2 \alpha)+0.5 s_{2} S i(4 \alpha)
$$




$$
+2 c^{2} \int_{0}^{2 \alpha} \frac{1-\cos u}{u} d u+\int_{0}^{2 \alpha} \frac{\sin ^{2} u\left(s^{2}-c^{2}\right)}{u} d u
$$

After completing some transformations and applying (34) to (35), we obtain the final expression for $R_{1}$ :

$$
\begin{gathered}
R 1=30 c^{2}\left[4 c^{2} C i_{n}(2 \alpha)-c_{2} C i_{n}(4 \alpha)\right. \\
\left.+s_{2} S i(4 \alpha)-s_{2} S i(2 \alpha)\right]
\end{gathered}
$$

Calculation of the second integral in (24) proceeds as follows, using (26), (25), (28) and (30) by turns:

$$
\begin{gathered}
\frac{R 2}{60 s_{2}}=\int_{0}^{\pi} \frac{[\cos (\alpha \cos \theta)-c][\sin (\alpha \cos \theta) \cos \theta-s]}{\sin \theta} d \theta \\
=\int_{-1}^{1} \frac{0.5 \sin (2 \alpha x) x-\operatorname{cs}(\alpha x) x}{1+x} d x-s \int_{-1}^{1} \frac{\cos (\alpha x)-c}{1+x} \\
=\int_{0}^{2 \alpha} \frac{0.5 \sin (2 u-2 \alpha)[(u / \alpha)-1]}{u} d u \\
-\int_{0}^{2 \alpha} \frac{c \sin (u-\alpha)[(u / \alpha)-1]}{u} d u-s \int_{0}^{2 \alpha} \frac{\cos (u-\alpha)-c}{u} d u \\
=c^{2} \int_{0}^{2 \alpha} \frac{\sin u}{u} d u-s\left(\int_{0}^{2 \alpha} \frac{\sin u}{u} d u-c \int_{0}^{2 \alpha} \frac{1-\cos u}{u} d u\right) \\
-\frac{c_{2}}{2} \int_{0}^{2 \alpha} \frac{\sin (2 u)}{u} d u+\frac{s_{2}}{2}\left(\int_{0}^{2 \alpha} \frac{\cos (2 u)}{u} d u-\int_{0}^{2 \alpha} \frac{\cos u}{u} d u\right) \\
=\left(-s^{2}+c^{2}\right) S i(2 \alpha)-0.5 c c_{2} S i(4 \alpha)+s_{2} C i_{n}(2 \alpha) \\
-0.5 s_{2} C i_{n}(4 \alpha)-s^{2} S i(2 \alpha)+0.5 s_{2} C i_{n}(2 \alpha)
\end{gathered}
$$

Thus, final expression for $R_{2}$ is

$$
\begin{aligned}
& R 2=15 s_{4}[2 \operatorname{Si}(2 \alpha)-\operatorname{Si}(4 \alpha)] \\
& 30 s_{2}^{2}\left[2 C i_{n}(2 \alpha)-C i_{n}(4 \alpha)\right]
\end{aligned}
$$

Calculation of the third integral in (24) proceeds as follows, using (7), (6), (9) and (11) by turns:

$$
\begin{aligned}
& \frac{R 3}{60 s^{2}}=\int_{0}^{\pi} \frac{[\sin (\alpha \cos \theta) \cos \theta-\mathrm{s}]^{2}}{\sin \theta} d \theta \\
& =\int_{-1}^{1} \frac{[\sin (\alpha x) x-\mathrm{s}]^{2}}{1+x} d x \\
& =\int_{0}^{2 \alpha} \frac{[\sin (u-\alpha)((u / \alpha)-1)-\mathrm{s}]^{2}}{u} d u
\end{aligned}
$$

$$
\begin{gathered}
=\frac{s^{2}}{\alpha^{2}} \int_{0}^{2 \alpha} u \cos ^{2} u d u-\frac{s_{2}}{2 \alpha^{2}} \int_{0}^{2 \alpha} u \sin (2 u) d u \\
\frac{c^{2}}{\alpha^{2}} \int_{0}^{2 \alpha} u \sin ^{2} u d u+\frac{2 s^{2}}{\alpha} \int_{0}^{2 \alpha}(1-\cos u) \cos u d u \\
-\frac{s_{2}}{\alpha} \int_{0}^{2 \alpha}(1-\cos u) \sin u d u+\frac{s_{2}}{2 \alpha} \int_{0}^{2 \alpha} \sin (2 u) d u \\
-\frac{2 c^{2}}{\alpha} \int_{0}^{2 \alpha} \sin ^{2} u d u+s^{2} \int_{0}^{2 \alpha} \frac{(1-\cos u)^{2}}{u} d u \\
+s_{2} \int_{0}^{2 \alpha} \frac{(1-\cos u) \sin u}{u} d u+\frac{c^{2}}{2} \int_{0}^{2 \alpha} \frac{1-\cos (2 u)}{u} d u \\
=1+\frac{1}{2 \alpha}\left(s_{2} c_{4}-s_{4} c_{2}\right)-2+\frac{s_{2}}{\alpha}+s_{2} S i(2 \alpha) \\
-0.5 s_{2} S i(4 \alpha)+2 s^{2} C_{n}(2 \alpha)+0.5 c_{2} C i_{n}(4 \alpha)
\end{gathered}
$$

Thus, final expression for $R_{3}$ is

$$
\begin{gathered}
R 3=-60 s^{2}+60 s^{2} s_{2} S i(2 \alpha)-30 s^{2} s_{2} S i(4 \alpha) \\
+120 s^{4} C i_{n}(2 \alpha)+30 s^{2} c_{2} C i_{n}(4 \alpha)+30 s^{2} s_{2} / \alpha
\end{gathered}
$$

Summarize expressions (17), (19) and (20) according to (5), performing all possible simplifications, the closed-form expression of (2) can be written as

$$
\begin{aligned}
R_{r}= & 30\left[3 \gamma+\ln \left(4 \alpha^{3}\right)+C i(4 \alpha)-4 C i(2 \alpha)\right. \\
& \left.+\sin ^{2} \alpha((\sin (2 \alpha) / \alpha)-2)\right]
\end{aligned}
$$

\section{Appendix II}

Euler's formula for the complex exponential will be used during the integration of (18):

$$
\begin{gathered}
e^{j x}=\cos (x)+j \sin (x), \sin (x)=\left(e^{j x}-e^{-j x}\right) / 2 j, \\
\cos (x)=\left(e^{j x}+e^{-j x}\right) / 2
\end{gathered}
$$

In order to apply this formula to shorten the expression of $Z_{m}$ (18), following constants should be introduced:

$$
k_{0}=15\left(e^{j k l}-e^{-j k l}\right) / j k, k_{1}=15\left(e^{j k l}+e^{-j k l}\right)
$$

then (18) can be written as

$$
Z_{m}=\int_{0}^{l}\left\{k _ { 0 } \left(\frac{e^{-j k R 1(z)}(z-l)(1+j k R 1(z))}{R 1^{3}(z)}\right.\right.
$$




$$
\begin{aligned}
& \left.-\frac{e^{-j k R 2(z)}(z+l)(1+j k R 2(z))}{R 2^{3}(z)}\right) \\
& -k_{1}\left(\frac{e^{-j k R 1(z)}}{R 1(z)}+\frac{e^{-j k R 2(z)}}{R 2(z)}\right) \\
& \left.+60 \frac{e^{-j k R 0(z)}}{R 0(z)}\right\}\left(e^{j k z}-e^{-j k z}\right) d z
\end{aligned}
$$

The integral $Z_{m}$ in (41) will be written as the sum of three integrals:

$$
Z_{m}=Z 1_{m}+Z 2_{m}+Z 3_{m}
$$

expressions for these terms are given below:

$$
\begin{gathered}
Z 1_{m}=\int_{0}^{l}\left[k_{0} \frac{(z-l)(1+j k R 1(z))}{R 1^{3}(z)}\right. \\
\times\left(e^{-j k(R 1(z)-z)}-e^{-j k(R 1(z)+z)}\right) \\
\left.-k_{1} \frac{e^{-j k(R 1(z)-z)}-e^{-j k(R 1(z)+z)}}{R 1(z)}\right] d z \\
\left(2_{m}=\int_{0}^{l}\left[k_{0} \frac{(z+l)(1+j k R 2(z))}{R 2^{3}(z)}\right.\right. \\
\left.-k_{1} \frac{\left.e^{-j k(R 2(z)+z)}-e^{-j k(R 2(z)-z)}\right)}{R 2(z)}\right] d z \\
Z 3_{m}=60 \int_{0}^{l} \frac{e^{-j k(R 0(z)-z)}-e^{-j k(R 0(z)+z)}}{R 0(z)} d z
\end{gathered}
$$

We introduce supplementary functions, defined as:

$$
\begin{gathered}
E R_{+}(z)=e^{-j k(R 1(z)+z),} \\
E R_{-}(z)=e^{-j k(R 1(z)-z)}
\end{gathered}
$$

Using (46) together with the constant $k_{2}=15(\exp (j k l)-\exp (-j k l))$, we will rewrite (43) as

$$
\begin{gathered}
Z 1_{m}=\int_{0}^{l}\left[k_{0} \frac{(z-l)\left(E R_{-}(z)-E R_{+}(z)\right)}{R 1^{3}(z)}\right. \\
+k_{2} \frac{(z-l)\left(E R_{-}(z)-E R_{+}(z)\right)}{R 1^{2}(z)}-k_{1} \frac{E R_{-}(z)-E R_{+}(z)}{R 1(z)}
\end{gathered}
$$

$$
\begin{gathered}
=k_{0} A_{0}+\int_{0}^{l}\left[k_{2} \frac{(z-l)\left(E R_{-}(z)-E R_{+}(z)\right)}{R 1^{2}(z)}\right. \\
\left.-k_{1} \frac{E R_{-}(z)-E R_{+}(z)}{R 1(z)}\right] d z
\end{gathered}
$$

The integral

$$
A_{0}=\int_{0}^{l} \frac{(z-l)\left(E R_{-}(z)-E R_{+}(z)\right)}{R 1^{3}(z)} d z
$$

from (47) will be integrated by parts using the well-known formula

$$
A_{0}=\int_{0}^{l} U(z) d V(z)=U(l) V(l)-U(0) V(0)-\int_{0}^{l} V(z) d U(z)(48)
$$

so the functions $U(z), V(z)$ and its derivatives must be found:

$$
\begin{gathered}
U(z)=\frac{E R_{-}(z)-E R_{+}(z)}{R 1^{2}(z)}, d V(z)=\frac{z-l}{R 1(z)} d z, \\
V(z)=\int d V(z)=R 1(z), d U(z)=\left[j k \frac{E R_{-}(z)+E R_{+}(z)}{R 1^{2}(z)}\right. \\
\left.+\left(E R_{+}(z)-E R_{-}(z)\right)\left(j k \frac{z-l}{R 1^{3}(z)}+2 \frac{z-l}{R 1^{4}(z)}\right)\right] d z
\end{gathered}
$$

Thus, after the substitution of (49) into (48) the integral $A_{0}$ will be written as

$$
\begin{gathered}
A_{0}=\frac{e^{-j k(a-l)}-e^{-j k(a+l)}}{a}-\frac{e^{-j k \sqrt{a^{2}+l^{2}}}}{\sqrt{a^{2}+l^{2}}} \\
-\frac{e^{-j k \sqrt{a^{2}+l^{2}}}}{\sqrt{a^{2}+l^{2}}}-\int_{0}^{l} R 1(z)\left[j k \frac{E R_{-}(z)+E R_{+}(z)}{R 1^{2}(z)}\right. \\
\left.+\left(E R_{+}(z)-E R_{-}(z)\right)\left(j k \frac{z-l}{R 1^{3}(z)}+2 \frac{z-l}{R 1^{4}(z)}\right)\right] d z
\end{gathered}
$$

After introducing the constant

$$
k_{3}=\frac{e^{-j k a}\left(e^{j k l}-e^{-j k l}\right)}{a}
$$

we will continue to record

$$
A_{0}=k_{3}-\int_{0}^{l}\left[2 \frac{(z-l)\left(E R_{+}(z)-E R_{-}(z)\right)}{R 1^{3}(z)}\right.
$$




$$
\begin{gathered}
\left.+j k \frac{(z-l)\left(E R_{+}(z)-E R_{-}(z)\right)}{R 1^{2}(z)}+j k \frac{E R_{-}(z)+E R_{+}(z)}{R 1(z)}\right] d z \\
=k_{3}+2 A_{0}-\int_{0}^{l}\left[j k \frac{(z-l)\left(E R_{+}(z)-E R_{-}(z)\right)}{R 1^{2}(z)}\right. \\
\left.+j k \frac{E R_{-}(z)+E R_{+}(z)}{R 1(z)}\right] d z
\end{gathered}
$$

The integral $A_{0}$ can be expressed from (50) as cyclical:

$$
\begin{aligned}
A_{0}=-k_{3} & +\int_{0}^{l}\left[j k \frac{(z-l)\left(E R_{+}(z)-E R_{-}(z)\right)}{R 1^{2}(z)}\right. \\
& \left.+j k \frac{E R_{-}(z)+E R_{+}(z)}{R 1(z)}\right] d z
\end{aligned}
$$

Substituting (51) into (47), we obtain

$$
\begin{gathered}
Z 1_{m}=k_{0}\left(-k_{3}+\int_{0}^{l}\left[j k \frac{(z-l)\left(E R_{+}(z)-E R_{-}(z)\right)}{R 1^{2}(z)}\right.\right. \\
\left.\left.+j k \frac{E R_{-}(z)+E R_{+}(z)}{R 1(z)}\right] d z\right)+\int_{0}^{l}\left[k_{2} \frac{(z-l)}{R 1^{2}(z)}\right. \\
\left.\times\left(E R_{-}(z)-E R_{+}(z)\right)-k_{1} \frac{E R_{-}(z)-E R_{+}(z)}{R 1(z)}\right] d z \\
=-k_{0} k_{3}+\int_{0}^{l}\left[\frac{(z-l)\left(E R_{+}(z)-E R_{-}(z)\right)}{R 1^{2}(z)}\left(k_{2}-k_{2}\right)\right. \\
+\frac{k_{2}\left(E R_{-}(z)+E R_{+}(z)\right)}{\left.R 1(z)+\frac{k_{1}\left(E R_{+}(z)-E R_{-}(z)\right)}{R 1(z)}\right]=k_{4}} \\
+30 \int_{0}^{l} \frac{e^{-j k(R 1(z)+(z-l))}-e^{-j k(R 1(z)-(z-l))}}{R 1(z)} d z
\end{gathered}
$$

where yet another constant is defined as

$$
k_{4}=-15 \frac{e^{-j k a}\left(e^{j k l}-e^{-j k l}\right)^{2}}{j k a}
$$

One can see that the expression (52) of the integral $Z 1_{m}$ significantly simpler than (43). After performing a similar simplification of (44), the result will be substituted into (42) together with (45) and (52), and the process of integration will be continued.

At first, sign of the integration variable in (44) will be changed ( $z \rightarrow-z)$; applying (46) and the obvious equality $R 2( \pm z)=R 1(\mp z)$, we rewrite (44) as

$$
\begin{gathered}
Z 2_{m}=-\int_{0}^{-l}\left[k_{0} \frac{(-z+l)(1+j k R 1(z))}{R 1^{3}(z)}\right. \\
\times\left(e^{-j k(R 1(z)-z)}-e^{-j k(R 1(z)+z)}\right) \\
\left.-k_{1} \frac{e^{-j k(R 1(z)+z)}-e^{-j k(R 1(z)-z)}}{R 1(z)}\right] d z \\
=\int_{0}^{l}\left[k_{0} \frac{(z-l)(1+j k R 1(z))\left(E R_{-}(z)-E R_{+}(z)\right)}{R 1^{3}(z)}\right. \\
\left.-k_{1} \frac{E R_{-}(z)-E R_{+}(z)}{R 1(z)}\right] d z=\int_{0}^{-l}\left[k_{0} \frac{(z-l)}{R 1^{3}(z)}\right. \\
\times\left(E R_{-}(z)-E R_{+}(z)\right)+k_{2} \frac{(z-l)\left(E R_{-}(z)-E R_{+}(z)\right)}{R 1^{2}(z)} \\
\left.-k_{1} \frac{E R_{-}(z)-E R_{+}(z)}{R 1(z)}\right] d z=k_{0} A_{1}+\int_{0}^{-l}\left[k_{2} \frac{(z-l)}{R 1^{2}(z)}\right. \\
\left.\times\left(E R_{-}(z)-E R_{+}(z)\right)-k_{1} \frac{E R_{-}(z)-E R_{+}(z)}{R 1(z)}\right] d z
\end{gathered}
$$

The integral

$$
A_{1}=\int_{0}^{-l} \frac{(z-l)\left(E R_{-}(z)-E R_{+}(z)\right)}{R 1^{3}(z)} d z
$$

from (53) will be integrated by parts using the well-known formula

$$
\begin{gathered}
A_{1}=\int_{0}^{-l} U(z) d V(z)=U(-l) V(-l)-U(0) V(0) \\
\quad-\int_{0}^{-l} V(z) d U(z)
\end{gathered}
$$

Substituting (49) into (54), we obtain

$$
\begin{gathered}
A_{1}=\frac{e^{-j k\left(\sqrt{a^{2}+4 l^{2}}+l\right)}-e^{-j k\left(\sqrt{a^{2}+4 l^{2}}-l\right)}}{\sqrt{a^{2}+4 l^{2}}} \\
-\frac{e^{-j k \sqrt{a^{2}+l^{2}}}-e^{-j k \sqrt{a^{2}+l^{2}}}}{\sqrt{a^{2}+l^{2}}}
\end{gathered}
$$

$$
-\int_{0}^{-l} R 1(z)\left[j k \frac{E R_{-}(z)+E R_{+}(z)}{R 1^{2}(z)}+\left(E R_{+}(z)-E R_{-}(z)\right)\right.
$$




$$
\left.\times\left(j k \frac{z-l}{R 1^{3}(z)}+2 \frac{z-l}{R 1^{4}(z)}\right)\right] d z
$$

After introducing the constant

$$
k_{5}=\frac{e^{-j k \sqrt{a^{2}+4 l^{2}}}\left(e^{-j k l}-e^{j k l}\right)}{\sqrt{a^{2}+4 l^{2}}}
$$

we will continue to record:

$$
\begin{gathered}
A_{1}=k_{5}+2 \int_{0}^{-l} \frac{(z-l)\left(E R_{-}(z)-E R_{+}(z)\right)}{R 1^{3}(z)} d z \\
-\int_{0}^{-l}\left[j k \frac{(z-l)\left(E R_{+}(z)-E R_{-}(z)\right)}{R 1^{2}(z)}+j k \frac{E R_{-}(z)+E R_{+}(z)}{R 1(z)}\right] d z \\
=k_{5}+2 A_{1}-\int_{0}^{-l}\left[j k \frac{(z-l)\left(E R_{+}(z)-E R_{-}(z)\right)}{R 1^{2}(z)}\right. \\
\left.+j k \frac{E R_{-}(z)+E R_{+}(z)}{R 1(z)}\right] d z
\end{gathered}
$$

The integral $A_{1}$ can be expressed from (55) as cyclical

$$
\begin{array}{r}
A_{1}=-k_{5}+\int_{0}^{-l}\left[j k \frac{(z-l)\left(E R_{+}(z)-E R_{-}(z)\right)}{R 1^{2}(z)}\right. \\
\left.+j k \frac{E R_{-}(z)+E R_{+}(z)}{R 1(z)}\right] d z
\end{array}
$$

Substituting (56) into (53), we obtain

$$
\begin{gathered}
Z 2_{m}=k_{0}\left(-k_{5}+\int_{0}^{-l}\left[j k \frac{(z-l)\left(E R_{+}(z)-E R_{-}(z)\right)}{R 1^{2}(z)}\right.\right. \\
\left.\left.+j k \frac{E R_{-}(z)+E R_{+}(z)}{R 1(z)}\right] d z\right)+\int_{0}^{-l}\left[k_{2} \frac{(z-l)}{R 1^{2}(z)}\right. \\
\left.\times\left(E R_{-}(z)-E R_{+}(z)\right)-k_{1} \frac{E R_{-}(z)-E R_{+}(z)}{R 1(z)}\right] d z \\
=-k_{0} k_{5}+\int_{0}^{-l}\left[\frac{(z-l)\left(E R_{+}(z)-E R_{-}(z)\right)}{R 1^{2}(z)}\left(k_{2}-k_{2}\right)\right. \\
\left.+\frac{k_{2}\left(E R_{-}(z)+E R_{+}(z)\right)}{R 1(z)}+\frac{k_{1}\left(E R_{+}(z)-E R_{-}(z)\right)}{R 1(z)}\right] d z=k_{6} \\
+30 \int_{0}^{-l} \frac{e^{-j k(R 1(z)+(z-l))}-e^{-j k(R 1(z)-(z-l))}}{R 1(z)} d z \quad(57)
\end{gathered}
$$

where yet another constant is defined as

$$
k_{6}=15 \frac{e^{-j k \sqrt{a^{2}+4 l^{2}}}\left(e^{j k l}-e^{-j k l}\right)^{2}}{j k \sqrt{a^{2}+4 l^{2}}}
$$

Substituting (52), (57) and (45) into (42), we obtain

$$
\begin{gathered}
Z_{m}=Z 1_{m}+Z 2_{m}+Z 3_{m}=k_{4} \\
+30 \int_{0}^{l} \frac{e^{-j k(R 1(z)+(z-l))}-e^{-j k(R 1(z)-(z-l))}}{R 1(z)} d z+k_{6} \\
+30 \int_{0}^{-l} \frac{e^{-j k(R 1(z)+(z-l))}-e^{-j k(R 1(z)-(z-l))}}{R 1(z)} d z \\
+60 \int_{0}^{l} \frac{e^{-j k(R 0(z)-z)}-e^{-j k(R 0(z)+z)}}{R 0(z)} d z \\
+\int_{0}^{l} \frac{\int_{0}^{-j} \frac{e^{-j k(R 1(z)+(z-l))}}{R 1(z)} d z-\int_{0}^{l} \frac{e^{-j k(R 1(z)-(z-l))}}{R 1(z)} d z}{R 1(z)} d z-\int_{0}^{-l} \frac{e^{-j k(R 1(z)-(z-l))}}{R 1(z)} d z \\
+2 \int_{0}^{l} \frac{\left.e^{-j k(R 0(z)-z)} d z-2 \int_{0}^{l} \frac{e^{-j k(R 0(z)+z)}}{R 0(z)} d z\right)+k 7}{R 0(z)}(58)
\end{gathered}
$$

where yet another constant is defined as

$$
k_{7}=60 \sin ^{2}(k l)\left(\frac{e^{-j k a}}{j k a}-\frac{e^{-j k \sqrt{a^{2}+4 l^{2}}}}{j k \sqrt{a^{2}+4 l^{2}}}\right)
$$

The following transformations will be applied to the six integrals, written under the bracket in (58):

1) the change of the integration variable will be performed in the first two integrals: $x=z-l$, $d x=d z$, after recalculation of the limits of integration we get: the upper integration limit $a=l-l=0$, the lower limit $b=0-l=-l$; then the equation

$$
R 0(\mathrm{z})=\left.\sqrt{a^{2}+x^{2}}\right|_{x=z-l}=\sqrt{a^{2}+(z-l)^{2}}=R 1(z)
$$

will be used for changing $R 1(z)$ on $R 0(z)$;

2) the inversion of the sign of the integration variable $(z \rightarrow-z)$ will be performed in the third and fourth integrals, after that the equation $R 2( \pm z)=R 1(\mp z)$ will be used. As a result of these transformations (58) will be written as

$$
Z_{m}=30\left(\int_{-l}^{0} \frac{e^{-j k(R 0(x)+x)}}{R 0(x)} d x-\int_{-l}^{0} \frac{e^{-j k(R 0(x)-x)}}{R 0(x)} d x\right.
$$




$$
\begin{aligned}
& -\int_{0}^{l} \frac{e^{-j k(R 2(z)+(-z-l))}}{R 2(z)} d z+\int_{0}^{l} \frac{e^{-j k(R 2(z)-(-z-l))}}{R 2(z)} d z \\
& \left.+2 \int_{0}^{l} \frac{e^{-j k(R 0(z)-z)}}{R 0(z)} d z-2 \int_{0}^{l} \frac{e^{-j k(R 0(z)+z)}}{R 0(z)} d z\right)+k_{7} \\
& =30\left(\int_{0}^{l} \frac{e^{-j k(R 2(z)+(z+l))}}{R 2(z)} d z-\int_{0}^{l} \frac{e^{-j k(R 2(z)-(z+l))}}{R 2(z)} d z\right. \\
& -\int_{0}^{-l} \frac{e^{-j k(R 0(x)+x)}}{R 0(x)} d x-\int_{0}^{l} \frac{e^{-j k(R 0(x)+x)}}{R 0(x)} d x \\
& \left.-2 \int_{0}^{-l} \frac{e^{-j k(R 0(z)+z)}}{R 0(z)} d z-2 \int_{0}^{l} \frac{e^{-j k(R 0(z)+z)}}{R 0(z)} d z\right)+k_{7} \\
& =30\left(\int_{0}^{l} \frac{e^{-j k(R 2(z)+(z+l))}}{R 2(z)} d z-\int_{0}^{l} \frac{e^{-j k(R 2(z)-(z+l))}}{R 2(z)} d z\right. \\
& \left.-3 \int_{0}^{-l} \frac{e^{-j k(R 0(z)+z)}}{R 0(z)} d z-3 \int_{0}^{l} \frac{e^{-j k(R 0(z)+z)}}{R 0(z)} d z\right)+k_{7}
\end{aligned}
$$

To find the closed-form expression of the self-impedance $Z_{m}$, we must transform (59) at first in order to reduce calculations to the exponential integral:

$$
E(z)=\int_{z}^{\infty} \frac{e^{-j u}}{u} d u=-C i(z)+j\left(S i(z)-\frac{\pi}{2}\right)
$$

where the functions $\mathrm{Si}(z)$ and $\mathrm{Ci}(z)$ are defined as (32) and (33) respectively. Integration with (60) will be implemented using

$$
\int_{b}^{a} \frac{e^{-j u}}{u} d u=E(b)-E(a)
$$

The following transformations will be applied to the four integrals, written under the bracket in (59):

1) the change of the variable in the first integral:

$$
\begin{gathered}
u=k(R 2(z)+(z+l)), d u=k\left(\frac{z+l}{R 2(z)} d z+d z\right) \\
=k\left(\frac{R 2(z)+(z+l)}{R 2(z)}\right) d z, \frac{d u}{u}=\frac{d z}{R 2(z)}
\end{gathered}
$$

after recalculation of the limits of integration we get: the upper limit

$$
a 1=k\left(\sqrt{a^{2}+4 l^{2}}+2 l\right)
$$

the lower limit

$$
b 1=k\left(\sqrt{a^{2}+l^{2}}+l\right)
$$

2) the change of the variable in the second integral:

$$
\begin{aligned}
u & =k(R 2(z)-(z+l)), d u=k\left(\frac{z+l}{R 2(z)} d z-d z\right) \\
& =-k\left(\frac{R 2(z)-(z+l)}{R 2(z)}\right) d z, \frac{d u}{u}=-\frac{d z}{R 2(z)}
\end{aligned}
$$

after recalculation of the limits of integration we get: the upper limit

$$
a 2=k\left(\sqrt{a^{2}+4 l^{2}}-2 l\right)
$$

the lower limit

$$
b 2=k\left(\sqrt{a^{2}+l^{2}}-l\right)
$$

3) the change of the variable in the third and fourth integrals:

$$
\begin{aligned}
u= & k(R 0(z)+z), d u=k\left(\frac{z}{R 0(z)} d z+d z\right) \\
= & k\left(\frac{R 0(z)+z}{R 0(z)}\right) d z, \frac{d u}{u}=\frac{d z}{R 0(z)}
\end{aligned}
$$

after recalculation of the limits of integration for the third integral we get: the upper limit

$$
a 3=k\left(\sqrt{a^{2}+l^{2}}-l\right)
$$

the lower limit $b 3=k a$; after recalculation of the limits of integration for the fourth integral we get: the upper limit

$$
a 4=k\left(\sqrt{a^{2}+l^{2}}+l\right)
$$

the lower limit $b 1=k a$.

After performing these transformations, we will rewrite (59) using (61):

$$
\begin{gathered}
Z_{m}=30\left(\int_{b 1}^{a 1} \frac{e^{-u}}{u} d u+\int_{b 2}^{a 2} \frac{e^{-u}}{u} d u-3 \int_{b 3}^{a 3} \frac{e^{-u}}{u} d u\right. \\
\left.-3 \int_{b 4}^{a 4} \frac{e^{-u}}{u} d u\right)+k_{7}=30\left[E\left(k\left(\sqrt{a^{2}+l^{2}}+l\right)\right)\right. \\
-E\left(k\left(\sqrt{a^{2}+4 l^{2}}+2 l\right)\right)+E\left(k\left(\sqrt{a^{2}+l^{2}}-l\right)\right) \\
\left.-E\left(k\left(\sqrt{a^{2}+4 l^{2}}-2 l\right)\right)\right]-3\left[E(k a)-E\left(k\left(\sqrt{a^{2}+l^{2}}-l\right)\right)\right]
\end{gathered}
$$




$$
-3\left[E(k a)-E\left(k\left(\sqrt{a^{2}+l^{2}}+l\right)\right)\right]
$$

After expressing the real $R_{m}$ and the imaginary $X_{m}$ parts of self-impedance $Z_{m}$ (62) according to (60), they can be written as

$$
\begin{aligned}
& R_{m}=60\left\{\operatorname { s i n } ^ { 2 } ( k l ) \left(\frac{\sin \left(k l \sqrt{4+(a / l)^{2}}\right)}{\left.k l \sqrt{4+(a / l)^{2}}-\frac{\sin (k a)}{k a}\right)+3 C i(k a)}\right.\right. \\
& +0.5 C i\left[k l\left(\sqrt{4+(a / l)^{2}}+2\right)\right]+0.5 C i\left[k l\left(\sqrt{4+(a / l)^{2}}-2\right)\right] \\
& \left.-2 C i\left[k l\left(\sqrt{1+(a / l)^{2}}+1\right)\right]-2 C i\left[k l\left(\sqrt{1+(a / l)^{2}}-1\right)\right]\right\} \\
& X_{m}=60\left\{\sin ^{2}(k l)\left(\frac{\cos \left(k l \sqrt{4+(a / l)^{2}}\right)}{k l \sqrt{4+(a / l)^{2}}}-\frac{\cos (k a)}{k a}\right)-3 \operatorname{Si}(k a)\right. \\
& -0.5 \operatorname{Si}\left[k l\left(\sqrt{4+(a / l)^{2}}+2\right)\right]-0.5 \operatorname{Si}\left[k l\left(\sqrt{4+(a / l)^{2}}-2\right)\right] \\
& \left.+2 \operatorname{Si}\left[k l\left(\sqrt{1+(a / l)^{2}}+1\right)\right]+2 \operatorname{Si}\left[k l\left(\sqrt{1+(a / l)^{2}}-1\right)\right]\right\}(64)
\end{aligned}
$$

\section{Acknowledgements}

This work was supported in part by the R.U. Ministry of Education and Science under Grant No. 716672011 and by the Novosibirsk State Technical University under Grant No. 028-NSG-13.

\section{REFERENCES}

[1] N. Kaneda, W. R. Deal, Y. Qian, R. Waterhouse, and T. Itoh, "A broad-band planar quasi-Yagi antenna," IEEE Trans. Antennas Propag., vol. 50, no. 8, pp. 1158-1160, Aug. 2002.

[2] G. S. Shiroma and W. A. Shiroma, "A two-element L-band quasi-Yagi antenna array with omnidirectional coverage," IEEE Trans. Antennas Propag., vol. 55, no. 12, pp. 3713-3716, Dec. 2007.

[3] S. E. Melais and T. M. Weller, "A quasi-Yagi antenna backed by a metal reflector," IEEE Trans. Antennas Propag., vol. 56, no. 12 , pp. $3868-3872$, Dec. 2008.

[4] J. A. Tirado-Mendez, H. Jardon-Aguilar, R. Flores-Leal, M. Reyes-Ayala, and F. Iturbide-Sanchez, "Inductively-loaded Yagi-Uda antenna with cylindrical cover for size reduction at VHF-UHF bands," IEEE Trans. Antennas Propag., vol. 59, no. 2, pp. 357-362, Feb. 2011.

[5] C. A. Balanis, Antenna Theory. Analysis and Design. Hoboken, NJ: John Wiley \& Sons, 2005.

[6] R. W. P. King, The Theory of Linear Antennas. Harvard University Press, 1956, p. 266, p. 392.

[7] L. J. Chu and J. A. Stratton, "Forced oscillations of a prolate spheroid," Journal of Applied Physics, vol. 12, no. 3, pp. 241-248, Mar. 1941.

[8] R. K. Mongia, I. J. Bahl, P. Bhartia, and J. Hong, RF and Microwave Coupled-Line Circuits. Norwood, MA: Artech House, 2007.

[9] E. B. Kaldjob, B. Geck, and H. Eul. "Impedance measurement of properly excited small balanced antennas," IEEE Antennas and Wireless Propagation Letters, vol. 8, pp. 193-195, 2009.

[10] J. D. Kraus, Antennas. New York: McGraw-Hill, 1950, pp. 426-428.

[11] B. M. Kolundzija, J. S. Ognjanovic, T. K. Sarkar. WIPL-D: Microwave Circuit and 3D EM Simulation for RF \& Microwave Applications. Software and User's Manual. Norwood, MA: Artech House, 2005. 